\title{
POLARIMETRIC CALIBRATION AND ASSESSMENT OF GF-3 IMAGES IN STEPPE
}

\author{
Y. Chang a ${ }^{\text {a }}$ J. Yang ${ }^{\text {a }}$, P. Li ${ }^{\text {a, }}{ }^{*}$, L. Shi ${ }^{\text {a }}$, L. Zhao ${ }^{\text {b, c }}$ \\ ${ }^{a}$ State Key Laboratory of Information Engineering in Surveying, Mapping and Remote Sensing, Wuhan University, China, 430079, \\ changyonglei@whu.edu.cn \\ ${ }^{\mathrm{b}}$ School of Remote Sensing and Information Engineering, Wuhan University, China, 430079, zhaolingli@whu.edu.cn \\ ${ }^{c}$ Beijing Key Laboratory of Urban Spatial Information Engineering, China, 100038
}

\section{Commission III, ICWG III/Iva}

KEY WORDS: Polarimetric calibration; PolSAR; GF-3; image quality; reflection symmetry

\begin{abstract}
:
The GaoFen-3 (GF-3) satellite is the first fully polarimetric synthetic aperture radar (PolSAR) satellite in China. It has three fully polarimetric imaging modes and is available for many applications. The system has been taken on several calibration experiments after the launch in Inner Mongolia by the Institute of Electronics, Chinese Academy of Sciences (IECAS), and the polarimetric calibration (PolCAL) strategy of GF-3 are also improved. Therefore, it is necessary to assess the image quality before any further applications. In this paper, we evaluated the polarimetric residual errors of GF-3 images that acquired in July 2017 in a steppe site. The results shows that the crosstalk of these images varies from $-36 \mathrm{~dB}$ to $-46 \mathrm{~dB}$, and the channel imbalance varies from $-0.43 \mathrm{~dB}$ to $0.55 \mathrm{~dB}$ with angle varying from -1.6 to 3.6 degree. We also made a PolCAL experiment to restrain the polarimetric distortion afterwards, and the polarimetric quality of the image got better after the PolCAL processing.
\end{abstract}

\section{INTRODUCTION}

The GF-3 satellite is the first fully polarimetric synthetic aperture radar (PolSAR) satellite in China. It has 12 imaging modes, including StripMap mode, ScanSAR mode, Spotlight mode, and ultra-fine strip mode. Furthermore, it has three PolSAR imaging modes, which are full-polarimetric strip I, full-polarimetric strip II, and wave imaging mode. Therefore, the satellite is available for many research and applications, and its designed applications are marine monitoring, disaster assistance, water monitoring, and meteorological observation. To carry on quantitative research and geophysical parameters inversion, polarimetric calibration (PolCAL) is requisite for PolSAR images. Since the launch of the satellite, the Institute of Electronics, Chinese Academy of Sciences (IECAS) has made several ground verifications in Inner Mongolia, and upgraded the calibration strategy of GF-3. Therefore, it is necessary to assess the image quality before any further researches or applications.

There are dozens of operational microwave types for fully polarimetric mode of GF-3, and the PolCAL situation of each wave type may be different. We can assess the polarimetric quality of some imaging wave types with the images that we acquired in steppes of Inner Mongolia. Although there are several new algorithms lately in many aspects (Shi, 2014, Villa, 2015, Souissi, 2016), the PolCAL methods based on reflection symmetry (Quegan, 1994) and scattering reciprocity (Ainsworth, 2006) are widely used in current PolSAR systems. To assess the polarimetric quality of images, methods based on manmade calibrators (Freeman, 1990, Whitt, 1991) are most accurate. In this study, we assessed the residual errors of GF-3 images by calculating the polarimetric distortion matrices (PDMs) with manmade calibrators and distributed targets.
Subsequently, we carried on PolCAL experiment on the GF-3 standard images to restrain the residual errors.

\section{METHODS}

\subsection{The Polarimetric Distortion Model}

During the observation, the scattering matrices of ground objects that observed by PolSAR systems are mixed with polarimetric errors. These errors includes the crosstalk, the copol channel imbalance, the cross-pol channel imbalance, and the system noise. Generally, the errors are expressed in PDMs by the PolSAR distortion model, which denotes that the real scattering matrices of ground objects are transformed into observed scattering matrices. And the model is (Quegan, 1994) :

$$
\begin{gathered}
\left(\begin{array}{cc}
O_{h h} & O_{h v} \\
O_{v h} & O_{v v}
\end{array}\right)=A\left[\begin{array}{ll}
1 & w \\
u & 1
\end{array}\right]\left[\begin{array}{ll}
k & 0 \\
0 & 1
\end{array}\right]\left(\begin{array}{ll}
S_{h h} & S_{h v} \\
S_{v h} & S_{v v}
\end{array}\right) * \\
{\left[\begin{array}{ll}
k & 0 \\
0 & 1
\end{array}\right]\left[\begin{array}{ll}
\alpha & 0 \\
0 & 1
\end{array}\right]\left[\begin{array}{ll}
1 & z \\
v & 1
\end{array}\right]+\left[\begin{array}{ll}
N_{h h} & N_{h v} \\
N_{v h} & N_{v v}
\end{array}\right]}
\end{gathered}
$$

In the model, $S_{h h}, S_{h v}, S_{v h}, S_{v v}$ compose the true scattering matrix of the ground object, with each of them representing a polarization channel. $O_{h h}, O_{h v}, O_{v h}, O_{v v}$ compose the observation matrix. The $u, w, v, z$ parameters are the crosstalk distortions. The $k$ term is the like-pol channel imbalance error, and $\alpha$ is the cross-pol channel imbalance error. Argument $A$ is the absolute calibration factor that will be used in the radiometric calibration. Letters $N_{h h}, N_{h v}, N_{v h}, N_{v v}$ represent the system noise in different channels.

* Corresponding author

PingxiangLi (pxli@whu.edu.cn) 


\subsection{Scattering Properties of PolCAL Reference Targets}

The landform of experiment site is steppe. The scattering mechanism of sparse grassland obeys the reflection symmetry attribute. This means that the objects are symmetric by the line of sight in radiometric scattering and the coherence of the likepolarization (like-pol) channel and cross-polarization (cross-pol) channel in their average covariance matrix is neglected. Apart from some particular manmade targets, most scattering media conform to the reciprocity property, i.e. $S_{h v}=S_{v h}$, which leads to the following form of covariance matrix (Ainsworth, 2006) :

$$
[C]=\left[\begin{array}{cccc}
C_{h h h h} & 0 & 0 & C_{h h v v} \\
0 & \beta & \beta^{\prime} & 0 \\
0 & \beta^{\prime} & \beta & 0 \\
C_{v v h h} & 0 & 0 & C_{v v v v}
\end{array}\right]
$$

in which the superscript $*$ is the complex conjugate operator, the values of $\beta$ and $\beta^{\prime}$ are real, and the others are complex. Since reciprocity implies that the two cross-pol channels are identical, for calibrated data, all the four correlation terms of the cross-pol channel must in fact be identical if the system noise is neglected. Based on these particular attributes, Quegan proposed a unified PolCAL method (Quegan, 1994), which is widely used in the current calibration processing of PolSAR images.

During the image acquisition, there were some polarimetric active radar calibrators (PARC) installed in this test site. There are three kind of PARCS, and the scattering matrices are:

$$
\begin{aligned}
& S_{x}=A_{x}\left[\begin{array}{ll}
0 & 1 \\
0 & 0
\end{array}\right], \quad S_{y}=A_{y}\left[\begin{array}{ll}
0 & 0 \\
1 & 0
\end{array}\right], \\
& S_{z}=A_{z}\left[\begin{array}{cc}
1 & 1 \\
e^{i \pi} & e^{i \pi}
\end{array}\right]
\end{aligned}
$$

The method using PARCs can get more accurate results but is only valid at a small place where the calibrators are placed. Therefore, both of the polarimetric methods that based on scattering symmetry and PARCs are deployed to evaluate the polarimetric errors.

\section{EXPERIMENTS}

The polarimetric calibration of GF-3 is a cyclical procedure, and the images within the same period and operational wave type are calibrated by the same PDMs. In this PolCAL strategy, the real PDMs of images within the same period should be similar, otherwise the images will still be distorted after PolCAL. Considering this, we assess the PDMs of images to verify the consistency, and the metadata of these images are listed below. The experiment site was in the Inner Mongolia. The terrain of the site is flat and far away from habitation. Meanwhile, the land cover of the site is mostly sparse grassland, making it a perfect field for PolCAL.

\subsection{Polarimetric Residual Error in the GF-3 Images}

The residual polarimetric errors are calculated with Quegan method, and the results are showed below. The polarimetric errors, i.e., the amplitude of cross-polarization channel imbalance (Alpha Amplitude), phase of cross-polarization channel imbalance (Alpha Phase), and the amplitude of crosstalk (Crosstalk Amplitude) are evaluated.

\begin{tabular}{cccc}
\hline $\begin{array}{c}\text { Acquisition } \\
\text { date }\end{array}$ & Image ID & $\begin{array}{c}\text { Central } \\
\text { look angle }\end{array}$ & $\begin{array}{c}\text { Wave } \\
\text { code }\end{array}$ \\
\hline \multirow{2}{*}{$2017-06-24$} & 2441191 & 43.92 & 208 \\
\cline { 2 - 4 } & 2441190 & 43.91 & 208 \\
\hline \multirow{2}{*}{$2017-07-06$} & 2463755 & 47.45 & 213 \\
\cline { 2 - 4 } & 2463754 & 47.44 & 213 \\
\hline \multirow{2}{*}{$2017-07-11$} & 2475549 & 40.01 & 203 \\
\cline { 2 - 4 } & 2475550 & 40.01 & 203 \\
\hline \multirow{2}{*}{$2017-07-16$} & 2491702 & 30.43 & 195 \\
\hline & 2491701 & 30.43 & 195 \\
\hline
\end{tabular}

Table 1. Parameters of the experimental GF-3 images

Currently, the satellite PolSAR systems use Transmit and Receive Modules (TRM) to control the processing microwave (Shimada, 2011). The images with the same wave code will have alike attributes. From the residual errors showed in Fig 1 and Fig 2 we can see that the images with the same wave code have similar polarimetric residual error, and they also have consistency in different image range.

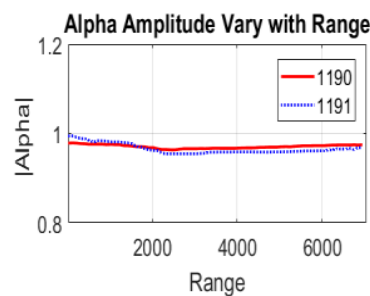

(a)

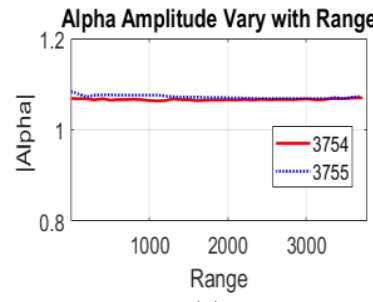

(c)

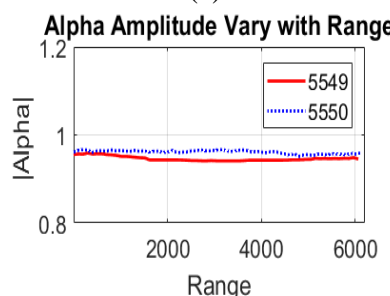

(e)

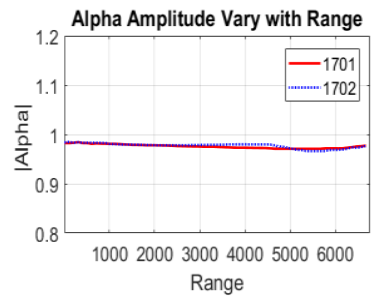

(g)

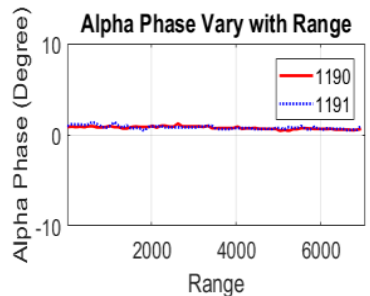

(b)

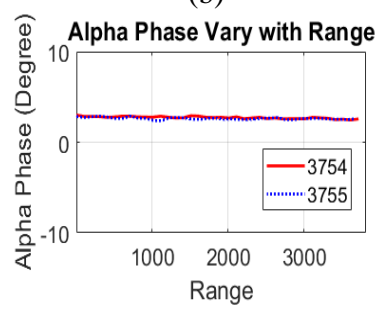

(d)

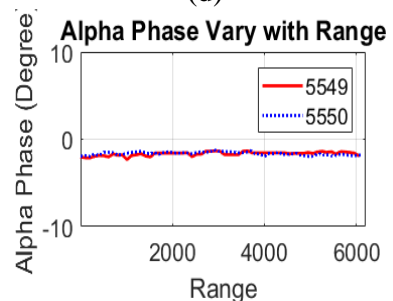

(f)

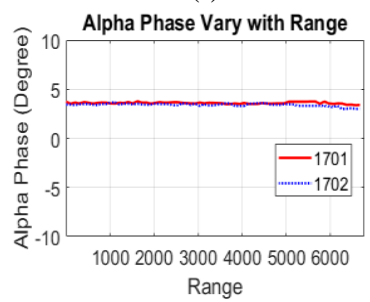

(h)
Figure 1. The residual Alpha Amplitude and Alpha Phase error in the image. The legend denotes the image number. Figure (a), (c), (e), (g) show the Alpha Amplitude error; Figure (b), (d), (f), (h) show the Alpha Phase error. 


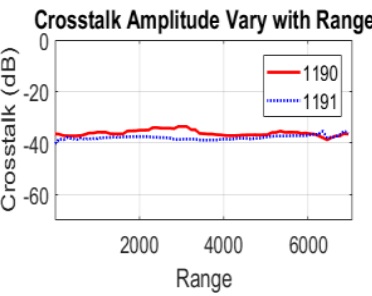

(a)

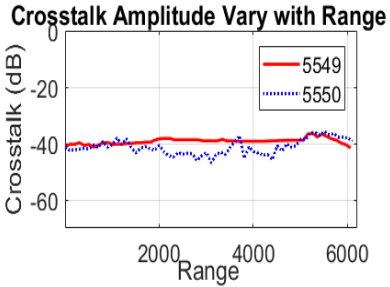

(c)

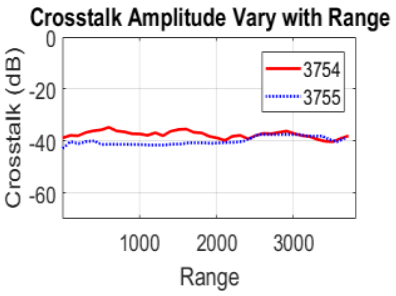

(b)

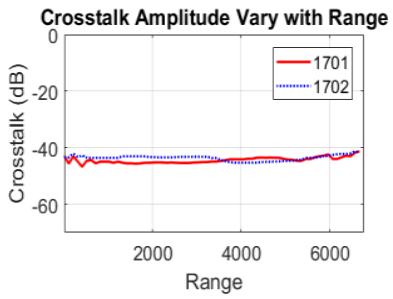

(d)
Figure 2. The residual Crosstalk Amplitude error in the image.

\begin{tabular}{cccc}
\hline $\begin{array}{c}\text { Image } \\
\text { ID }\end{array}$ & $\begin{array}{c}\text { Crosstalk } \\
(\mathbf{d B})\end{array}$ & $\begin{array}{c}|\boldsymbol{\alpha}| \\
(\mathbf{d B})\end{array}$ & $\begin{array}{c}\operatorname{Arg}(\boldsymbol{\alpha}) \\
(\mathbf{d e g} .)\end{array}$ \\
\hline $\mathbf{2 4 4 1 1 9 1}$ & $-37 \pm 4.0$ & $-0.28 \pm 0.15$ & $0.8 \pm 0.3$ \\
\hline $\mathbf{2 4 4 1 1 9 0}$ & $-36 \pm 2.9$ & $-0.22 \pm 0.04$ & $0.8 \pm 0.2$ \\
\hline $\mathbf{2 4 6 3 7 5 5}$ & $-40 \pm 3.8$ & $0.55 \pm 0.19$ & $2.6 \pm 0.1$ \\
\hline $\mathbf{2 4 6 3 7 5 4}$ & $-38 \pm 2.4$ & $0.48 \pm 0.01$ & $2.7 \pm 0.1$ \\
\hline $\mathbf{2 4 7 5 5 4 9}$ & $-39 \pm 4.3$ & $-0.43 \pm 0.07$ & $-1.6 \pm 0.4$ \\
\hline $\mathbf{2 4 7 5 5 5 0}$ & $-41 \pm 3.3$ & $-0.30 \pm 0.03$ & $-1.6 \pm 0.2$ \\
\hline $\mathbf{2 4 9 1 7 0 2}$ & $-43 \pm 4.1$ & $-0.16 \pm 0.06$ & $3.5 \pm 0.4$ \\
\hline $\mathbf{2 4 9 1 7 0 1}$ & $-46 \pm 3.9$ & $-0.18 \pm 0.03$ & $3.6 \pm 0.1$ \\
\hline
\end{tabular}

Table 2. Polarization residual errors after PolCAL

To give a quantitative results, we calculated the average residual errors along the image range, see in Table 2 . The results shows that the crosstalk of these images varies from $36 \mathrm{~dB}$ to $-46 \mathrm{~dB}$, and the channel imbalance varies from $-0.43 \mathrm{~dB}$ to $0.55 \mathrm{~dB}$ with angle varying from -1.6 to 3.6 degree.

\subsection{PolCAL Refinement Test on the Image}

To suppress the residual polarimetric errors in the GF-3 images and refine the image quality, we carried on a PolCAL test. We calibrate the image by Quegan PolCAL method with polarimetric parameters that showed in the previous section. To verify the effect of PolCAL, we compared the polarimetric errors of the images before and after PolCAL. We chose Freeman PolCAL method that based on PARCs to calculate the polarimetric errors, which is more objective.

There are three PARCs that satisfy the formulas in section 2.2 on the image 2441191. So we carried PolCAL test on this image. To compare the polarization error conveniently, the parameters calculated by Freeman PolCAL method were transformed into the format of Quegan PolCAL method. The experiment result is in Table 3. The results indicate that the polarization errors calculated by Quegan method and Freeman method are a little different. Nevertheless, the Quegan PolCAL can still improve the polarimetric quality of the image. We can see that the channel imbalance was suppressed to an acceptant level, while the crosstalk changed little, which were already in a fine accuracy before PolCAL.

\section{CONCLUSION}

In this paper, we presented an experiment on the polarization quality of GF-3 images in a steppe site. Eight images acquired in July 2017 were tested. The results indicates that the images with the same wave code have similar polarimetric residual error and are the errors are consistent in image range. The crosstalk of these images varies from $-36 \mathrm{~dB}$ to $-46 \mathrm{~dB}$, and the channel imbalance varies from $-0.43 \mathrm{~dB}$ to $0.55 \mathrm{~dB}$ with angle varying from -1.6 to 3.6 degree. We also showed an image PolCAL experiment in this paper, and the polarimetric residual errors were suppressed to a preferable level after the PolCAL. Generally, the calibration program of GF-3 is still in progress, and the image quality of this satellite may get better over time.

\begin{tabular}{cccccccccc}
\hline & \multicolumn{4}{c}{ Crosstalk Amplitude(dB) } & \multicolumn{4}{c}{ Channel Imbalance } \\
\hline W & $\mathbf{U}$ & $\mathbf{V}$ & $\mathbf{Z}$ & $|\boldsymbol{\alpha}|(\mathbf{d B})$ & $\mathbf{A r g}(\boldsymbol{\alpha})(\mathbf{d e g})$. & $|\mathbf{k}|(\mathbf{d B})$ & $\operatorname{Arg}(\mathbf{k})(\mathbf{d e g})$. \\
\hline $\begin{array}{c}\text { Before PolCAL } \\
\text { Quegan }\end{array}$ & -48.2 & -39.0 & -43.9 & -37.8 & -0.40 & 0.82 & -0.05 & -5.37 \\
\hline $\begin{array}{c}\text { Before PolCAL } \\
\text { Freeman }\end{array}$ & -47.8 & -40.6 & -54.0 & -36.1 & -0.29 & 3.71 & -0.39 & -7.54 \\
\hline $\begin{array}{c}\text { After PolCAL } \\
\text { Freeman }\end{array}$ & -38.9 & -43.8 & -45.9 & -44.9 & 0.07 & 2.33 & -0.30 & -1.83 \\
\hline
\end{tabular}

Table 3. Polarization errors before and after PolCAL

\section{ACKNOWLEDGEMENTS}

The research was founded by the National Natural Science Foundation of China, Grant No. 41771377, No. 41501382, and No. 41601355, also funded by Beijing Key Laboratory of Urban Spatial Information Engineering, under Grant 2017215. The authors thank the China Centre for Resources Satellite Data and Application and the Institute of Electronics, Chinese Academy of Sciences for providing the valuable data.

\section{REFERENCES}

Ainsworth, T. L., L. Ferro-Famil and J.-S. Lee (2006). "Orientation angle preserving a posteriori polarimetric SAR calibration." IEEE Trans. Geosci. Remote Sens 44(4): 994-1003.

Freeman, A., Y. Shen and C. L. Werner (1990). "Polarimetric SAR calibration experiment using active radar calibrators." IEEE Trans. Geosci. Remote Sens 28(2): 224-240.

Quegan, S. (1994). "A unified algorithm for phase and crosstalk calibration of polarimetric data-theory and observations." IEEE Trans. Geosci. Remote Sens 32(1): 89-99. 
Shi, L., J. Yang and P. Li (2014). "Co-polarization channel imbalance determination by the use of bare soil." ISPRS $J$. Photogram. Remote Sens 95: 53-67.

Shimada, M. (2011). "Model-based polarimetric SAR calibration method using forest and surface-scattering targets." IEEE Trans. Geosci. Remote Sens 49(5): 1712-1733.

Souissi, B. and M. Ouarzeddine (2016). "Polarimetric SAR Data Correction and Terrain Topography Measurement Based on the Radar Target Orientation Angle." Journal of the Indian Society of Remote Sensing: 1-15.

Villa, A., L. Iannini, D. Giudici, A. Monti-Guarnieri and S. Tebaldini (2015). "Calibration of SAR Polarimetric Images by Means of a Covariance Matching Approach." IEEE Trans. Geosci. Remote Sens 53(2): 674-686.

Whitt, M. W., F. T. Ulaby, P. Polatin and V. V. Liepa (1991). "A general polarimetric radar calibration technique." IEEE Trans. Antenn. Propa. 39(1): 62-67. 